components of the bacterial immune system other than CRISPR-Cas, such as restriction enzymes or other antiviral defence systems ${ }^{9}$.

Many questions remain to be answered concerning the bacterial immunosuppression generated by anti-CRISPR proteins. How long will a bacterium remain in this defenceless state? How does this state vary from cell to cell in a bacterial population? Although this cooperation strategy seems to provide one way for phages to neutralize CRISPR-Cas complexes, perhaps other strategies remain to be discovered that do not require the sacrifice of the first wave of infecting phages.

Aude Bernheim and Rotem Sorek are in the Department of Molecular Genetics, Weizmann Institute of Science, Rehovot 76100, Israel. e-mail:rotem.sorek@weizmann.ac.il

1. Borges, A. L. et al. Cell https://doi.org/10.1016/ j.cell.2018.06.013 (2018).

2. Landsberger, M. et al. Cell https://doi. org/10.1016/j.cell.2018.05.058 (2018).

3. Hille, F. et al. Cell 172, 1239-1259 (2018).

4. Borges, A. L., Davidson, A. R. \& Bondy-Denomy, J. Annu. Rev. Virol. 4, 37-59 (2017).

5. Pawluk, A., Davidson, A. R. \& Maxwell, K. L. Nature Rev. Microbiol. 16, 12-17 (2018).

6. Garneau, J. E. et al. Nature 468, 67-71 (2010).

7. Ptashne, M. A Genetic Switch 3rd edn (Cold Spring Harbor Laboratory Press, 2004).

8. Erez, Z. et al. Nature 541, 488-493 (2017).

9. Doron, S. et al. Science 359, eaar4120 (2018).

R.S. declares competing financial interests. See go.nature.com/216rtqk for details.

\title{
Histidine degradation boosts cancer therapy
}

\begin{abstract}
Clinical use of the anticancer drug methotrexate can be limited by its high toxicity. It emerges that a diet rich in the amino acid histidine increases the effectiveness of methotrexate treatment and lowers toxicity in mice. SEE LETTER P.632
\end{abstract}

\section{CHRISTIAN FREZZA}

$\mathrm{M}$ ethotrexate was one of the first approved anticancer drugs, and is a cornerstone of modern chemotherapy for the treatment of certain solid tumours and blood cancers. However, this therapy must often be stopped prematurely because of its high toxicity in patients' healthy cells. On page 632, Kanarek et al. ${ }^{1}$ report that degradation of the amino acid histidine can increase the sensitivity of cancer cells to methotrexate, and the results suggest that this approach can also help to lower the side effects of methotrexate treatment in mouse models.

An enzyme cofactor termed folate is required for DNA and protein synthesis. In 1948 , it was found ${ }^{2}$ that leukaemia is particularly sensitive to drugs that block folate synthesis. Methotrexate inhibits the enzyme dihydrofolate reductase (DHFR), which synthesizes the active form of folate known as tetrahydrofolate (THF) from its precursor dihydrofolate (DHF) (Fig. 1). However, when this drug is used in the clinic, it can substantially reduce the level of folate in healthy cells - a side effect that is only partially relieved by taking folate supplements. If this toxicity reaches a level that compromises the function of healthy cells, therapy may have to be stopped prematurely. There is therefore great interest in trying to reduce the side effects of methotrexate treatment.

To investigate the processes that affect the cellular response to methotrexate, Kanarek et al. used a cell line derived from a person with leukaemia. They employed a gene-editing technique that prevents the expression of individual genes throughout the entire genome in such cells, and tested whether a decrease in the expression of any particular gene reduced the cells' sensitivity to methotrexate. One such gene that they identified encodes a protein called SLC19A1, which transports folate
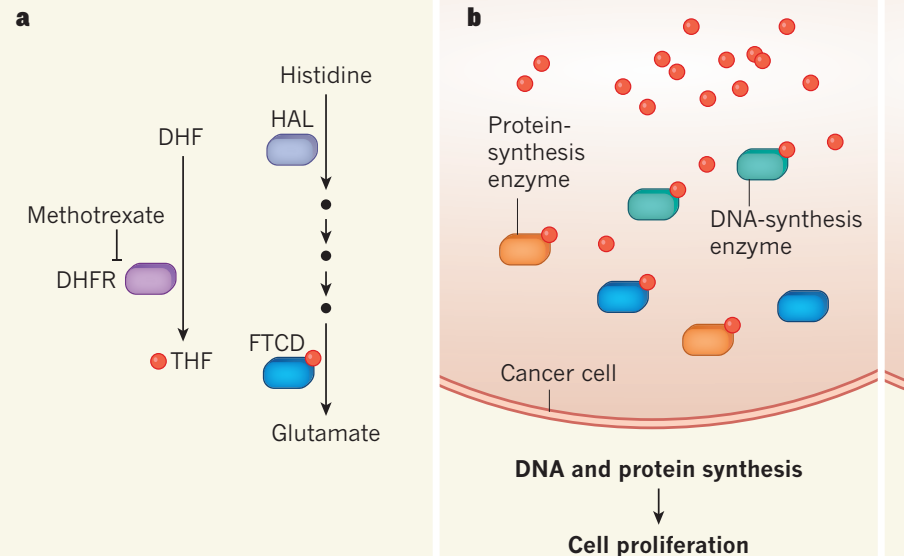

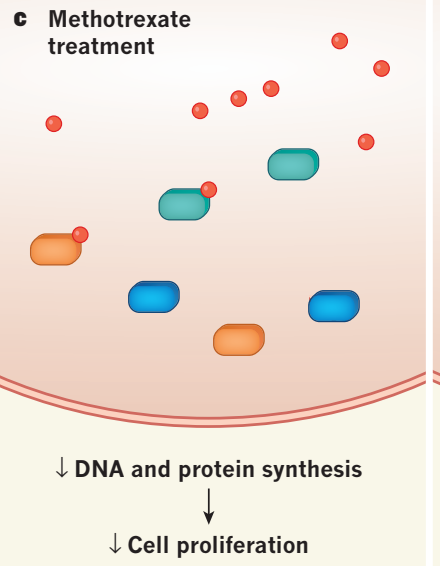

d Methotrexate + histidine treatment

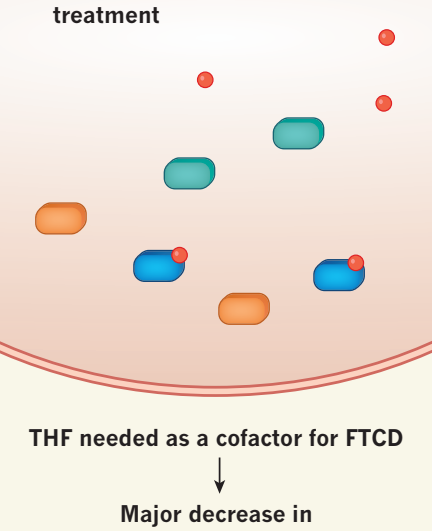

DNA and protein synthesis and in cell proliferation
Figure 1 The effectiveness of the cancer drug methotrexate can be improved by histidine degradation. a, The cofactor tetrahydrofolate (THF) is required for the function of enzymes, including those that catalyse processes needed for cell proliferation. The enzyme dihydrofolate reductase (DHFR) generates THF from dihydrofolate (DHF), and this catalytic reaction can be inhibited by the anticancer drug methotrexate. Kanarek et al. ${ }^{1}$ report that, in mouse models and human cell lines, the sensitivity of cancer cells to methotrexate can be boosted by the metabolic degradation of the amino acid histidine. Histidine is metabolized to the amino acid glutamate through a series of intermediate molecules (black circles) in a pathway that requires the enzyme histidine ammonia lyase (HAL), and, crucially for this effect on methotrexate treatment, the THF-dependent enzyme formimidoyltransferase cyclodeaminase (FTCD). b, In cancer cells, a large pool of THF is available for THF-dependent enzymes, such as FTCD or those needed for cell proliferation. c, In methotrexate-treated cancer cells, a decrease in THF levels reduces the activity of these enzymes and thereby reduces tumour growth. d, The authors report that tumour growth in mice given methotrexate and a histidine-rich diet was substantially decreased compared with $\mathbf{c}$. This might be because histidine degradation coopts THF for use with FTCD, thereby enhancing the effect of methotrexate. 
and methotrexate into cells. This result was unsurprising, given that SLC19A1 has previously been linked ${ }^{3}$ to sensitivity to methotrexate, but it confirmed the power of the authors' approach.

Kanarek and colleagues found that decreasing the expression of a gene that encodes the enzyme formimidoyltransferase cyclodeaminase (FTCD) also reduced the sensitivity of the cancer cells to methotrexate. FTCD degrades histidine, an amino acid that humans must obtain from their diet because the body cannot synthesize it. The authors reasoned that, because FTCD requires THF as a cofactor for its activity, a decrease in the intracellular levels of FTCD could help to limit the reduction of cellular pools of THF that occurs with methotrexate treatment. Consistent with this hypothesis, the authors found that when the levels of FTCD were reduced by gene editing, THF depletion in human cancer cells treated with methotrexate was less than that seen in methotrexate-treated cells in which FTCD levels were not reduced.

On the basis of these findings, the authors predicted that the manipulation of other steps in the histidine-degradation pathway could affect the efficacy of methotrexate treatment. To test this, they again used gene editing to decrease the expression of other genes in this pathway in human cancer cells grown in vitro. They found that such decreases did indeed mimic the effect of decreased FTCD levels in preserving the cellular pool of THF in methotrexate-treated cells.

The authors then analysed a panel of cancer cell lines, and observed a correlation between cell lines that had low expression of the enzyme histidine ammonia lyase (HAL), which acts at the first step in the histidinedegradation pathway, and low sensitivity to the effects of methotrexate. Moreover, when Kanarek and colleagues analysed data ${ }^{4}$ available for people who have a form of leukaemia that is commonly treated with methotrexate, they found that patients who had high levels of HAL expression had higher survival rates than those with low HAL expression levels. Perhaps this is because methotrexate is more effective when high levels of HAL boost histidine degradation and the depletion of THF pools by FTCD.

The authors therefore proposed that greater cellular histidine degradation should deplete the THF pool and thus increase the effectiveness of methotrexate. To explore this prediction, they generated tumours in mice, and gave the animals methotrexate and a histidine-rich diet. They treated the animals with lower doses of methotrexate than those normally used in such models, to assess whether histidine had a synergistic effect in this context. The histidine-rich diet did indeed boost tumour-growth inhibition in response to lowdose methotrexate compared with the effects of methotrexate in animals that did not receive a dietary histidine boost.

An unexpected and exciting finding of
Kanarek and colleagues' work is that, in their mouse models, the toxicity that is usually seen in non-cancerous tissues with methotrexate treatment did not occur when the animals were given a histidine-rich diet, despite the extra toxicity that would have been expected as a result of the depletion of THF in these tissues. The results not only indicate that histidine can increase methotrexate-driven cytotoxicity in cancer cells, but also raise the possibility that some mechanism is reducing the toxic effects of THF depletion in normal tissues. Do normal and tumour tissue differ in sensitivity in this context as a result of having different thresholds for toxicity, or is the rate of histidine degradation different in normal and tumour tissues?

Given the technical challenges involved in measuring certain intermediate molecules in the histidine-degradation pathway, histidine's contribution to the folate pool still needs to be quantified in vitro and in vivo to validate the authors' model. Furthermore, there are other pathways ${ }^{5}$, in addition to that of histidine degradation, for example degradation of the amino acid methionine, that can affect the THF level in cells. It will be important to investigate the extent to which such pathways contribute to the THF pool, and whether they also affect methotrexate-associated toxicity.

Kanarek and colleagues' work indicates the potential value of conducting clinical trials to investigate whether histidine supplementation can boost the effectiveness of methotrexate in cancer treatment. If this is the case, further clinical trials would be needed to determine the optimal dosage. Histidine supplementation might provide an inexpensive and safe strategy for increasing the efficacy of methotrexate. Supplementation with low doses does not have adverse effects in humans, although doses of more than 8 grams of histidine a day can lead to zinc deficiency ${ }^{6}$. However, there have been no extensive studies to investigate possible detrimental effects of a histidine-rich diet in humans.

Finally, these results might have relevance for people with rheumatoid arthritis or other chronic autoimmune diseases, in which lowdose methotrexate is the standard treatment ${ }^{7}$ and folate supplements are the only strategy currently available to limit side effects. Could a histidine-rich diet reduce side effects in these people also, and increase their well-being?

Christian Frezza is at the MRC Cancer Unit, University of Cambridge, Hutchison/MRC Research Centre, Cambridge CB2 OXZ, UK. e-mail:cf366@mrc-cu.cam.ac.uk

1. Kanarek, N. et al. Nature 559, 632-636 (2018).

2. Farber, S., Diamond, L. K., Mercer, R. D., Sylvester, R. F. Jr \& Wolff, J. A. N. Engl. J. Med. 238, 787-793 (1948).

3. Assaraf, Y. G. \& Schimke, R. T. Proc. Natl Acad. Sci. USA 84, 7154-7158 (1987).

4. Roberts, K. G. et al. N. Engl. J. Med. 371, 1005-1015 (2014).

5. Locasale, J. W. Nature Rev. Cancer 13, 572-583 (2013).

6. Institute of Medicine of the National Academies. Dietary Reference Intakes for Energy, Carbohydrate, Fiber, Fat, Fatty Acids, Cholesterol, Protein, and Amino Acids Ch. 10 (Natl Acad. Press, 2005).

7. Brown, P. M., Pratt, A. G. \& Isaacs, R. G. Nature Rev. Rheumatol. 12, 731-742 (2016)

This article was published online on 11 July 2018.

How the head governs
the heart of fly species

The males of two closely related species of fly respond differently to a female pheromone. It emerges that this difference is due to alterations in the activity of an evolutionarily conserved neural circuit in the brain. SEE ARTICLE P.564

\section{NICOLAS GOMPEL \\ \& BENJAMIN PRUD'HOMME}

$\mathrm{P}$ articular sensory signals - a certain odour or taste, for instance - can trigger opposing responses in different species. What changes in neural circuits could elicit such contradictory behaviours? On page 564, Seeholzer et al. ${ }^{1}$ compare the circuits that control courtship in males in two closely related species of fruit fly that behave differently when exposed to a particular pheromone. They find an explanation for these opposing behaviours that few neuroscientists would have predicted.
While wandering on a rotten apple, a male fruit fly is likely to encounter a variety of other flies, only a few of which will be females of the same species. Identifying and courting these conspecific females is a crucial step in securing the male fly's reproductive success. But this is not always an easy task. For instance, two sister species of fruit fly - Drosophila melanogaster and Drosophila simulans - are often found on the same fruits. Males of these species therefore rely heavily on sensing pheromones to identify conspecific females ${ }^{2}$.

To test whether a female fly is of the same species, the male touches her abdomen with 